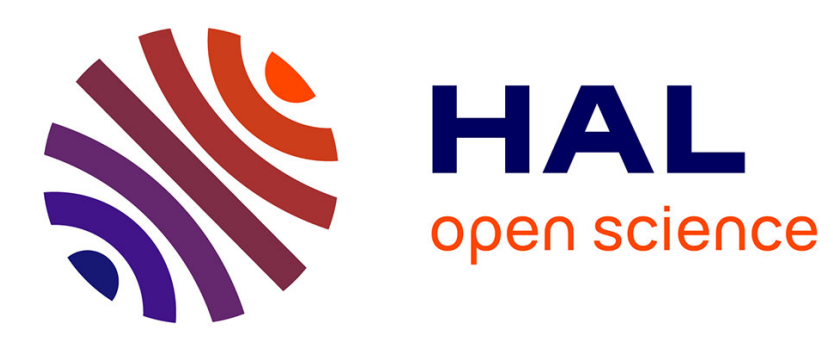

\title{
La crémation : des pratiques singulières à l'élaboration d'un cadre de référence
}

Gaëlle Clavandier

\section{To cite this version:}

Gaëlle Clavandier. La crémation: des pratiques singulières à l'élaboration d'un cadre de référence. Etudes sur la mort. Revue de la Société de thanatologie, 2007, 132, pp.65-86. halshs-00662585

\section{HAL Id: halshs-00662585 https://shs.hal.science/halshs-00662585}

Submitted on 26 Jan 2012

HAL is a multi-disciplinary open access archive for the deposit and dissemination of scientific research documents, whether they are published or not. The documents may come from teaching and research institutions in France or abroad, or from public or private research centers.
L'archive ouverte pluridisciplinaire HAL, est destinée au dépôt et à la diffusion de documents scientifiques de niveau recherche, publiés ou non, émanant des établissements d'enseignement et de recherche français ou étrangers, des laboratoires publics ou privés. 


\title{
LA CRÉMATION : DES PRATIQUES SINGULIĖRES À L'ÉLABORATION D'UN CADRE DE RÉFÉRENCE
}

\author{
Gaëlle CLAVANDIER
}

La présente réflexion porte sur le statut, tant légal que social, de la crémation en France. Si chacun d'entre nous a réfléchi au mode de sépulture qu'il lui conviendrait, ce choix n'est pas nécessairement effectif. Le sociologue perçoit une certaine indétermination à ce propos. Du reste, il n'est pas rare qu'un aller-retour s'opère entre inhumation et crémation. Ainsi, un assistant funéraire nous disait qu'il n'est pas insolite qu'une personne qui a souscrit à un testament obsèques, revienne le voir par la suite pour modifier les closes du contrat, la valse des hésitations pouvant s'étaler sur plusieurs années : «Moij'ai un gars, ça fait plus d'un an qu'ilhésite »(Gilbert). Un autre professionnel insiste également sur le poids de la «déclaration » à la famille, si elle a lieu; laquelle peut introduire un sentiment de doute chez le souscripteur d'un contrat d'obsèques : "lorsqu'elle a annoncé à sa fille qu'elle souhaitait se faire incinérer, celle-ci lui a dit qu'elle préférerait la voir inhumer, comme cela se fait traditionnellement dans la famille. La vieille dame est revenue me voir et a décidé de se faire inhumer» (Monique). S'il est patent qu'opter pour un mode de sépulture ou un autre, à savoir l'inhumation ou la crémation, est un droit ancien, puisqu'il émane de la loi du 15 novembre $1887^{1}$, l'évolution des pratiques actuelles est sans commune mesure avec cette déclaration de principe.

Si chaque personne a une idée de ce qu'est la crémation, y a-t-il possibilité aujourd'hui de proposer une définition univoque du «phénomène crématiste»? Rien n'est moins sûr, nous en voulant pour preuve les débats qui animent aujourd'hui, les professionnels (opérateurs funéraires), les pou-

1. Laquelle est relative à la liberté des funérailles. 
voirs publics et le législateur, mais aussi les «familles ». Les individus eux, de fait, sont de leur vivant face à une alternative, qui aura une incidence sur leur corps, sur les rituels funéraires, sur la localisation de la sépulture ou l'absence de celle-ci, sur le caractère public ou strictement intime du souvenir qui s'élaborera au fil des années, pour se défaire ou non.

Bien que très important, le point de vue des familles et du quidam sera en partie évacué de notre propos et étudié en d'autres lieux, afin de nous concentrer ici sur les acteurs institutionnels et professionnels qui font aujourd'hui évoluer la pratique crématiste; non pas en tant qu'alternative à l'inhumation mais dans ses conditions même (en terme de sépultures, de statut des cendres, de destinations possibles...). Si les pratiques et représentations de la crémation se construisent pas à pas, dans les usages quotidiens, il va de soi que le cadre proposé par les opérateurs funéraires et le législateur constituent une ossature formelle et symbolique.

\section{LA CRÉMATION, LES CRÉMATIONS...}

Rare sont les faits sociaux qui atteignent des taux de croissance telle la crémation. Autorisée en 1963 par l'Église catholique, la crémation n'en demeure pas moins une pratique quasi-inexistante jusque dans les années $1980^{2}$. Puis, elle se développe de manière significative avec dans un premier temps des taux de croissances annuels allant de $10 \%$ à plus de $20 \%$; des taux qui, de fait, diminuent à partir des années 1990 alors que le nombre des crémations est en augmentation constante ${ }^{3}$.

Il va de soi que cette question des modes de sépulture devient cruciale en raison de l'évolution statistiques de la pratique crématiste, qui concerne de nos jours un quart des décès. À partir du moment où l'usage est massif (en 2005, environ 110000 crémations ont été pratiquées) et lorsque les vœux affichés d'avoir recours à cette technique se multiplient ${ }^{4}$, il n'est plus possi-

2. Le taux de crémation est environ de $1 \%$ à cette date.

3. On dénombre 1755 crémations en 1970, 2338 en 1975, l'augmentation est régulière cependant le taux de crémation au regard des inhumations reste marginal. L'essor de la crémation est manifeste dans les années 1980. P. Belhassen prend l'exemple de l'année 1985 avec 14565 crémations soit une croissance de 23,3\% par rapport à l'année précédente mais un faible volume au regard du nombre total de décès, à savoir $2,64 \%$. Voir P. Belhassen, La crémation: le cadavre et la loi, Paris, L.G.D.J., Panthéon-Assas, 1997, p. 24-26.

4. Des estimations tendent à démontrer que près de $40 \%$ des contrats d'obsèques font mention d'une crémation. Sachant que ces contrats ont valeur testamentaire, il ne s'agit pas simplement d'un acte déclaratif. Ce chiffre nous a été communiqué à plusieurs reprises sur le terrain lors de notre enquête. Il est également conforme à celui notifié par l'association Colombe qui gère les volontés de près de 126000 personnes (contrat d'obsèques) dans son dossier de 
ble de percevoir cette démarche comme une intention atypique. Les prévisions de l'Association Française d'Information Funéraire anticipent que les crémations devraient s'établir à $35 \%$ en 2020, puis à $49 \%$ en 2030. L'équilibre emblématique, à savoir la moitié des sépultures, serait donc atteint dans moins d'un quart de siècle, vraie révolution en matière funéraire au regard des transformations lentes des siècles passés ${ }^{5}$.

Évidemment il ne s'agit pas d'établir une lecture quantitativiste d'un tel phénomène car cela n'aurait aucun sens vu la portée du geste. Exercer son libre arbitre sur ses propres funérailles n'est pas un acte anodin, pas plus qu'il ne serait indépendant de toutes normes, croyances, valeurs... Choisir la crémation participe d'une réelle démarche. Indubitablement, le temps des pionniers ${ }^{6}$, des sociétés et associations crématistes, libres penseurs comme ils aiment à se nommer, est révolu. Néanmoins, la crémation, si elle tend à devenir une pratique commune, n'en est pas moins en phase d'élaboration normative. Qu'est-ce que la crémation, outre le fait de réduire un corps à l'état de cendres? Il va de soi, que l'incinération telle qu'elle est pratiquée traditionnellement, en Inde notamment, avec ses bûchers, ses préconisations rituelles, ses obligations sociales, n'a rien à voir ou presque avec la crémation contemporaine dans les pays occidentaux. En outre, peut-on évoquer un phénomène singulier? Point de réponse évidente, car peut-on par exemple amalgamer des pratiques aussi disparates telles : l'inhumation de l'urne dans le caveau familial; son dépôt dans un columbarium ou un cavurne; la dispersion des cendres dans un lieu symbolique tel une forêt que le défunt aimait arpenter; la pose de l'urne sur le buffet familial; la dispersion au sortir du crématorium dans le «jardin du souvenir »? Sans parler des pratiques atypiques : performances artistiques, abandon de l'urne cinéraire, agrégation ou au contraire division des cendres en plusieurs contenants. Assurément, chacune de ces réalités a trait à la crémation - puisque le cadavre a été réduit en cendres dans un crématorium selon une technique réglementée et qu'une cérémonie a marqué le temps des adieux ${ }^{7}$ - cependant, les modes de souvenirs qu'elles supposent, la localisation de celui-ci, les

presse. Association fondée en 1998 à l'initiative de trois organismes professionnels (prévoyance funéraire: OGF, aide et service à domicile : ADMR et compagnie d'assurance: AMPHITEA). Pour autant, le nombre de contrats qui représentent environ $15 \%$ des cas n'est pas suffisant pour en tirer des conclusions définitives. En effet, on peut penser que les personnes qui ont fait le choix de la crémation souhaitent en faire une mention claire, afin que leurs volontés soient respectées.

5. Voir P. Ariès, Essais sur l'histoire de la mort en Occident du moyen âge à nos jours, Paris, Seuil, 1975, et L'homme devant la mort, Paris, Seuil, 1977. M. Vovelle, Mourir autrefois, Paris, Gallimard, 1974 et La mort en occident de 1300 à nos jours, Paris, Gallimard, 1983.

6. Voir V. Souffron, Brûler et non pourrir. Pour une socio-anthropologie de la crémation, Toulouse, Thèse de doctorat de sociologie, 1999.

7. Voir M. Hanus, «Le cadavre crématisé », Etudes sur la mort, n¹29, 2006, p. 139. 
valeurs qu'elles instituent sont finalement très différents. Parler de la crémation au singulier suppose de doter cette pratique funéraire d'un ensemble de valeurs, de recommandations et de référentiels communs, c'est ce à quoi s'est attelé le législateur, sachant qu'il atteste d'un mouvement plus ancien et plus global, d'un véritable changement sociétal.

Plus que de décrire les pratiques en émergence, celles de tout un chacun, cet article va dégager les nouveaux enjeux, les pistes de réflexions autour de la crémation, lesquels affectent très largement l'inhumation ${ }^{8}$. Pour cela nous proposons une réflexion en trois temps. Partant de la proposition de loi votée au Sénat le 22 juin $2006^{9}$ qui scelle la nécessité d'un débat sur la question, nous détaillerons ensuite le décret du 12 mars $2007^{10}$, qui s'il ne modifie guère la loi actuelle du 8 janvier 1993, a néanmoins entériné le fait que la crémation doit bénéficier d'un cadre légal plus strict. Les transformations réglementaires sont sans doute à venir et il est intéressant de noter dès à présent, comment les acteurs de terrain se saisissent de ces prémisses de changement. Pour cela nous avons recueilli les réactions des représentants des opérateurs funéraires ${ }^{11}$, ainsi que les réflexions des professionnels du secteur funéraire qui intègrent ces modifications légales à l'aune de leur activité ${ }^{12}$.

L'hypothèse est faite que plus que le choix de la crémation, c'est son environnement, ses conséquences, l'après du geste si l'on peut dire, qui pose problème aujourd'hui. La réflexion sur la destination des cendres, sur le statut de celles-ci, sur le garant (à savoir aujourd'hui «la personne ayant

8. Avoir un contrepoids, tel la crémation a pour conséquence de faire évoluer les pratiques d'inhumation. Il existe sur le marché du funéraire des «monuments mixtes » qui au-delà de la possibilité de sceller une urne sur un monument classique (tombe), envisagent un espace hybride où univers de l'inhumation et de la crémation se rejoignent. Certains édifices prennent la forme d'une tombe horizontale avec en arrière plan un columbarium à deux cases, d'autres jouent sur les référents symboliques dans un esprit syncrétique alliant tradition et mysticisme. Ainsi, l'Egypte ancienne à travers le Nil, où la tradition hindouiste avec la figure emblématique du Gange, viennent donner à l'inhumation un autre aspect que celui habituellement admis.

9. Cette proposition de loi fait suite à une proposition de loi de M. J.-P. Sueur relative à la législation funéraire, $\mathrm{n}^{\circ} 464$, déposée le 7 juillet 2005, laquelle a été renvoyée à la commission des lois constitutionnelle, de législation, du suffrage universel, du règlement et d'administration générale. La commission des lois saisie au fond a nommé J.-R. Lecerf rapporteur le 7 juin 2006 (rapport $n^{\circ} 386$ déposé le 13 juin 2006). La discussion en séance publique a eu lieu le 22 juin 2006 (TA $\left.{ }^{\circ} 111\right)$. La proposition de loi relative à la législation funéraire $\left(\mathrm{n}^{\circ} 3186\right)$ adoptée par le Sénat a été déposée à l'Assemblée Nationale le même jour et renvoyée à la commission des lois constitutionnelles.

10. Décret n²007-328 du 12 mars 2007 publié au JO du 13 mars 2007.

11. Ce terrain est de seconde main comme nous l'expliquerons par la suite.

12. Par le biais d'une enquête de terrain en cours. 
qualité pour pourvoir aux funérailles ») rend compte que la crémation n'est pas simplement un geste fait sur un corps sans vie. En effet, le droit de recourir à ce mode de « sépulture » est un droit inaliénable semble-t-il, cependant parler de mode de sépulture suppose de ne pas s'arrêter au traitement du corps/cadavre. La surprise de tenir en ses mains une urne encore chaude au sortir du crématorium, la difficulté de déterminer une destination pour les cendres, l'impossibilité parfois de se recueillir ensuite en un lieu défini, ne sont pas des problèmes négligeables ou tout au moins circonstanciés. Le geste crématiste dans toute sa portée suppose de réinterroger la place ${ }^{\mathbf{1 3}}$ que l'on souhaite, en tant que vivants, donner aux morts, de questionner le souvenir des morts $^{14}$ tel qu'il est traditionnellement pratiqué en France ${ }^{15}$. Cette nouvelle donne n'est pas fixée et c'est ce mouvement qui nous intéresse ici.

\section{ÉLÉMENTS JURIDIQUES ET RÉGLEMENTAIRES}

Au vu de cette évolution des usages et croyances, le législateur s'est saisi de ce réel bouleversement pour en faire l'objet d'une vaste réflexion relative à la législation funéraire. La proposition de loi du 22 juin 2006 adoptée en première lecture au Sénat ${ }^{16}$ aurait eu pour incidence de transformer radicalement l'activité des opérateurs funéraires, de promouvoir une politique de développement des sites cinéraires (exclusivement publics) et crématoriums et de donner une orientation sur les pratiques relatives à la crémation. Elle visait à mieux encadrer la crémation en œuvrant pour la reconnaissance du statut des cendres et en limitant les modes de conservation et de dispersion des cendres ; mais aussi à contrôler la qualification des opérateurs funéraires en améliorant notamment la formation professionnelle des personnels ; puis elle avait pour objectif de simplifier les démarches des familles en rendant effectives les dispositions prises.

13. En référence à l'ouvrage de P. Baudry, La place des morts. Enjeux et rites, Paris, Armand Colin, 1999.

14. En référence à l'ouvrage de J.-H. Déchaux, Le souvenir des morts - Essai sur le lien de filiation.

15. Toute une littérature sociologique a analysé la montée de la pratique crématiste à l'aune de la thèse du déni de la mort apparue dans les années 1970-1980. Voir en particulier, J.-D. Urbain, L'archipel des morts. Cimetières et mémoire en Occident, Paris, Petite bibliothèque Payot, 1998 (première édition 1989), du même auteur, «Mort traquée, mort tracée - Culte des morts, crémation, sida », Ethnologie française, vol. 28, 1998, p. 43-49; L.-V. Thomas, La mort en question - Traces de mort et mort des traces, Paris, l'Harmattan, 1991.

16. La loi devait être ensuite adoptée à l'assemblée Nationale. Dans un premier temps il a été question qu'elle soit examinée avant le $1^{\text {er }}$ novembre 2006, durant la session parlementaire. Puis son examen a été repoussé jusqu'au décret de mars 2007. 
Cette proposition de loi, si elle n'a pas encore vu le jour, n'en demeure pas moins un référentiel sur lequel il est nécessaire de s'appuyer, sachant que son vote au sénat a fait l'unanimité ${ }^{17}$. Le décret 2007-328 du 12 mars 2007 «relatif à la protection des cendres funéraires»ordonné par B. Hortefeux, ministre des Collectivités territoriales prend acte de certaines modifications prévues par la proposition de loi, tout en les pondérant. Qui plus est, étant centrée sur la protection des cendres et plus particulièrement sur la destination possible de celles-ci, il reste très relatif car les transformations semblent à venir plus qu'effectives. Comme tout décret, son dessein et de doter les pratiques d'un cadre plus explicite qu'en l'absence de celui-ci.

Première modification tangible, la famille notion large et polémique, puisqu'elle renvoie à une réalité vécue plus que légale, est remplacée par l'expression: «toute personne ayant qualité pour pourvoir aux funérailles ». Ainsi, jusqu'à aujourd'hui, une concubine ayant eu des enfants avec son compagnon peut légitimement se sentir appartenir à la «famille» de celui-ci, or elle n'avait aucun droit concernant ses funérailles, droit qui revenait dans ce cas prioritairement aux enfants du défunt, puis à ses petits-enfants et enfin à ses parents. Désormais, de son vivant, une personne peut faire mention de sa volonté qu'une personne soit mandatée pour pourvoir à ses funérailles.

Second bouleversement, sans doute celui qui supporte les changements à venir, s'il demeure possible de conserver une urne à titre privé ou de disperser les cendres en pleine nature, ces pratiques deviennent l'exception. En effet, le cimetière et plus précisément les sites cinéraires deviennent la règle. Il est précisé - de manière vague il faut en convenir - que sans mention faite par le défunt, la seule destination envisageable est le cimetière. Le texte se construit de la manière suivante:

- Le cadre est posé, «Ala demande de cette personne [personne qui a qualité pour pourvoir aux funérailles] qui justifie de son identité et de son domicile, soit l'urne est inhumée dans une sépulture, déposée dans une case de columbarium ou scellée sur un monument funéraire à l'intérieur d'un cimetière ou d'un site cinéraire prévu à l'article L. 2223-40, soit les cendres sont dispersées dans le lieu spécialement affecté à cet effet prévu à l'article R. 2223-9 ou un espace aménagé à cet effet d'un site cinéraire. Le dépôt ou l'inhumation de l'urne ou la dispersion des cendres dans un cimetière ou dans un site cinéraire sont effectués après autorisation du maire », en modification de l'article R. 2213-39 du code des collectivités territoriales.

17. Faute de place, nous renvoyons à la lecture de l'article de J.-P. Sueur, promoteur de la loi, dans le présent numéro. Ainsi, qu'à un article à paraître, Gaëlle Clavandier, «Quand le droit énonce la norme. Le cas de la crémation». 
- Puis vient l'exception : «Toutefois, si telle est la volonté exprimée par le défunt, soit l'urne est déposée ou inhumée dans une propriété privée, soit les cendres sont dispersées en pleine nature, sans pouvoir l'être sur les voies publiques, Le dépôt ou l'inhumation de l'urne ou la dispersion des cendres sont effectuées après déclaration auprès du maire de la commune du lieu de dépôt, d'inhumation de l'urne ou de la dispersion des cendres », en modification de l'article R. 2213-39 du code des collectivités territoriales.

Le caractère privatif (du dépôt ou de la dispersion), ainsi qu'une dis-

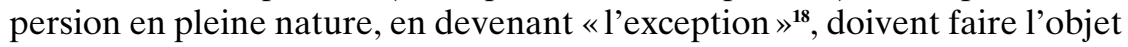
d'une attention particulière. Ainsi, troisième transformation notable, la traçabilité des cendres doit être patente. La remise de l'urne reste possible si le défunt en a fait la demande, mais elle doit dorénavant faire l'objet d'une déclaration auprès «du maire de la commune du lieu du dépôt ». Le texte règle de la sorte, au moins en partie, le problème du devenir de l'urne en cas de décès de celui qui en avait la charge, l'urne revient de droit au cimetière. L'alternative est identique lorsque la personne qui recueillie l'urne à son domicile souhaite se démettre de cette fonction.

B. Hortefeux indique dans un communiqué que ce décret a pour but de réformer le régime de la crémation et de "s'assurer de l'existence d'un minimum de garanties en matière de destination des cendres ». Des garanties qui viennent suppléer des comportements perçus comme déviants.

Les réactions ont été nombreuses suite au décret du 12 mars 2007. Bien évidemment, l'une des premières voix à s'être élevée est celle du promoteur de la proposition de loi du 22 juin 2006, J.-P. Sueur, lequel dit ne pas se contenter de cette première étape. Dès la parution du décret, il indique clairement, que celui-ci ne peut «se substituer à la proposition de loi qui a été adoptée, à [son] initiative, par le Sénat unanime le 22 juin dernier qui traite d'un grand nombre de questions relatives à la législation funéraire, et qui relèvent clairement de la loi ${ }^{19}$. En effet, le sénateur, émet l'avis qu'un décret n'est pas à la hauteur de l'enjeu, en ne traitant qu'essentiellement de la destination des cendres, la question du statut de celles-cin'est pas réglée. En outre, traiter de la destination avant même de doter les cendres d'un statut est aller trop vite en besogne. Plus avant, c'est la possible appropriation des cendres à titre privatif qui n'est pas tranchée, l'existence de sites cinéraires privés n'est pas réellement abordée et si la conservation ou la dispersion des cendres dans une propriété privée devient l'exception elle subsiste (tel

18. Exception ne voulant pas dire quantitative, mais qualitative dans la mesure où la cette pratique peut être répandue en volume mais perçue d'un point de vue légal comme dérogatoire.

19. Communiqué du 13 mars 2007. 
n'était pas le cas dans la proposition de loi). Bien évidemment, en se focalisant sur la protection des cendres, l'ensemble des autres dispositions est ici évacué.

\section{LES RÉACTIONS DES ORGANISATIONS PROFESSIONNELLES}

Ce décret s'il donne du grain à moudre aux juristes, n'en a pas moins une dimension applicative. Dès lors, les professionnels du secteur funéraire se sont emparés de cette disposition officielle liminaire pour faire entendre leurs voix. La particularité de cette parole est qu'elle marque un hiatus, lequel révèle une distance avec les textes légaux mais pas seulement. Les organisations professionnelles, syndicales, les fédérations et associations parlent en leur nom, parfois au nom de la profession ou du secteur funéraire mais ces voix sont discordantes. Sans faire ici une sociologie des professions et des organisations, nous allons nous concentrer sur le contenu de ce hiatus et voir quels sont néanmoins les points de convergence. Lorsqu'il s'agit de porter une critique sur le décret, tel qu'il vient d'être pris et plus largement sur les projets de loi tels qu'ils s'annoncent, la force du «terrain » s'arc-boute sur une connaissance intrinsèque face à des ordonnateurs qui eux statuent à partir de réflexions exogènes. La ratification de textes ayant une incidence sur les pratiques quotidiennes des opérateurs, a pour conséquence de faire émerger des avis, mais aussi de remobiliser la nécessité d'établir un corps intermédiaire qui viendrait dire sa spécificité, donc sa différence. Se pose d'emblée la question de la légitimité.

Certaines de ces réactions ont pris la forme de réponses publiques, dont plusieurs sont directement accessibles et non réservées à un cercle d'initiés. Nous nous sommes attachées à analyser celles publiées dans le cadre de la revue Reson@nce, laquelle est à destination des professionnels du funéraire $^{20}$. Le site Internet de la Revue permet de les consulter-et si cette consultation est peu effective deux mois après la mise en ligne ${ }^{21}$ - elle reste néanmoins un «témoin » de la vigilance des professionnels à l'égard du législateur. Ces commentaires sont intéressants à étudier dans la mesure où ils donnent une idée du climat actuel à propos de la crémation, chez les

20. Suite à la parution au Journal Officiel le 13 mars 2007, du décret relatif à la protection des cendres funéraires, Reson@nce «a souhaité interroger les personnalités représentatives de la profession », preuve de l'importance que revêtent les tentatives de réformer le droit funéraire pour les opérateurs. Voir http://www.resonnance-mag.com.

21. Chacun des avis donne lieu à une page consultable de manière autonome. Deux mois jour pour jour après leur publication, à savoir le 20 avril 2007, chaque page a été consultée d'une dizaine à une vingtaine de reprises. 
«décideurs». Les avis sont tranchés, la controverse est là.

Nous prenons le parti de penser que ces avis devront être amendés, retravaillés, à l'aune de la pratique quotidienne des professionnels sur le terrain. Nous nous appuierons donc dans un second temps sur une série d'entretiens auprès d'assistants funéraires et de maîtres de cérémonies qui côtoient quasi journellement la crémation.

Certes non représentative, l'analyse qui suit émane de personnalités reconnues du monde funéraire au sens large, la plupart étant des opérateurs ou des représentants de ceux-ci (syndicats, fédération, groupement d'entreprises), sans oublier la Fédération Française des Crématistes ${ }^{22}$. Faisant autorité, leur point de vue dépasse très largement une appréciation personnelle, pour rendre compte d'une opinion mainte fois discutée au sein des organisations en question. Les convictions, avis, préconisations, critiques, voire diatribes énoncées font date. D’un point de vue général deux aspects sont à retenir :

- d'une part, les professionnels se félicitent que le cimetière soit promulgué 《destination naturelle des cendres ${ }^{23}$, l'urne à domicile ou la dispersion en un lieu autre qu'un site cinéraire ou un cimetière devenant l'exception ${ }^{24}$;

- d'autre part, les personnes consultées expriment une réelle difficulté à se saisir et à mettre en œuvre le texte, qui en l'état ne satisfait quiconque (ni les tenants de la proposition de loi, ni ses détracteurs).

La difficulté à appliquer le décret est maintes fois dénoncée : « Le décret tel qu'il est paru est en effet difficile, sinon impossible, à interpréter et à

22. Le lecteur peut donc consulter les avis de : Jacques Cousin, Président de la Fédération Française de Crémation ; François Michaud-Nérard, Directeur Général des Services Funéraires Ville de Paris; Michel Marchetti et Michel Minard, Co-présidents de Confédération des Professionnels du Funéraire et de la Marbrerie; Gérard Véclin, de France Obsèques Liberté; Corinne Loïodice, Présidente de l'Union des Professionnels du Pôle Funéraire Public; Bernard Rolet, Président de l'Union Nationale de l'UPF; Philippe Gentil, Président du groupe ROCECLERC; Alain Hoffarth et Roger Schvartsman, Co-présidents de la Fédération Française de Pompes Funèbres ; Pierre Vidalet, Président de l'Union des Gestionnaires de Crématoriums Français; Patrick Cave, Directeur du crématorium et du parc du mémorial de la Vienne; Guillaume d'Abbadie, Co-auteur du code pratique des opérations funéraires; Thomas Beaucourt, Président de l'Association pour les Droits et Obligations des Crématoriums ; JeanPierre Sirugue, Président de VF Assistance. Notre propos n'est pas de discuter l'exhaustivité de la parole ici présentée, mais d'en rendre compte en ce qu'elle est rendue publique et sollicitée.

23. Selon les termes de F. Michaud-Nérard, Directeur Général des Services Funéraires de la Ville de Paris.

24. Nous reviendrons sur cette idée en conclusion car elle marque le passage de la règle à la norme, cette dernière devenant l'expression du «naturel». 
appliquer $»^{25}$. Il serait le signe patent d'un décalage entre les grandes orientations définies dans le cadre de la loi et les pratiques au quotidien des acteurs: «On peut cependant regretter le caractère complexe de ce nouveau texte au regard de la réalité quotidienne que vivent les familles en deuil $\gg^{26}$.

Toute une série de questions se pose, interrogations plurielles qui s'énoncent sur différents répertoires. Le décret concernant en particulier la destination des cendres, c'est la possible traçabilité qui est éprouvée: sur quel registre inscrire le mode de conservation ou de dispersion choisi? L'enregistrement doit-il être fait dans la commune du décès, dans la commune du crématorium, voire dans la commune sur le territoire où les cendres ont été dispersées ${ }^{27}$ ? Comment savoir ce que fera la famille en cas de remise de l'urne, respectera-t-elle les dispositions indiquées à l'opérateur funéraire ayant organisé les obsèques ? Qui doit faire la déclaration, l'opérateur funéraire, la famille? Nous le verrons, cet aspect est également un point critique de la réflexion des assistants funéraires.

Le rôle des entreprises de pompes funèbres, leurs obligations quant à la nature de la déclaration préalable faite par le défunt est également en suspend: qu'en est-il d'un défunt décédé accidentellement qui n'a pas fait de demande de crémation? Quelle forme doit prendre les volontés exprimées par le défunt, doivent-elles être écrites ? Les assistants funéraires ontils obligation d'informer les familles sur leur devoir de traçabilité concernant l'urne ? Qu'en est-il lorsque, après plusieurs années de conservation au domicile, les cendres sont répandues? Que se passe-t-il si une partie des cendres sont dispersées et une partie conservée? rien n'indique avec précision ce qui doit être fait lorsque le dépositaire des cendres décède à son tour ${ }^{28}$ ? Pas plus qu'on ne sait répondre avec assurance à la question de savoir qui est la personne «ayant qualité à pourvoir aux funérailles»?

Comme le mentionne F. Michaud-Nérard, Directeur Général des Services Funéraires de la Ville de Paris, le décret ne règle pas la question du statut des cendres et des sites cinéraires et ce de façon pratique: "Le cavurne ou la case de columbarium sont-ils considérés comme des sépultures? (...) L'espace de dispersion [le jardin du souvenir] est-il considérécomme une sépulture? Peut-il y avoir perception de taxes de dispersion en dehors des frais correspondant à l'acte technique de dispersion? $\gg^{29}$. Un de ses confrères, non

25. F. Michaud-Nérard, Directeur Général des Services Funéraires de la Ville de Paris.

26. P. Gentil, Président du groupe ROC-ECLERC.

27. Le décret précise que cette inscription doit se faire dans la commune où a eu lieu la dispersion, ou dans la commune où ont été déposées les cendres. Mais que se passe-t-il en cas de déménagement? En cas de sortie du territoire français? En cas de dispersion en pleine mer?

28. Si la destination «idéale» apparaît être le cimetière ou un site cinéraire, les cendres seront-elles automatiquement répandues dans le jardin du souvenir?

29. F. Michaud-Nérard, Directeur Général des Services Funéraires Ville de Paris. 
sans polémique, va plus loin en invalidant l'énoncé même du décret: "Contrairement à son intitulé: "Décret relatif à la protection des cendres funéraire”, il nous semble que ce décret ne protégera que l'urne, et non les cendres $»^{30}$ ! Du même coup, dans cette perspective, le décret devient une coquille vide, puisque c'est le contenant qui est protégé, un objet, non les cendres qui auraient pu, à cette occasion, être instituées « restes humains».

Afin, de lever les incertitudes inhérentes à la formulation même du texte, et de définir comment chacun à son niveau doit s'en saisir, nombres de professionnels demandent une circulaire d'application, et certains souhaitent mener, dans leurs organismes, des consultations auprès de leurs adhérents. Au final, c'est l'image d'un texte imprécis et mal adapté, "une mesurette »" qui prédomine chez les représentants des professionnels du funéraire. Lesquels voient dans ce décret une demande accrue de formalités administratives, sans avoir pour autant réglé les problèmes relatifs à la crémation. Cependant, il ne faut pas se méprendre, les précisions attendues par tous n'impactent en rien le bien fondé ou non de cette démarche ${ }^{32}$. Il faut donc faire une distinction entre la philosophie du texte et son mode d'application.

Plus largement, la publication de ce décret a eu pour conséquence une réflexion sur les enjeux de la profession, son devenir et son implication dans la redéfinition actuelle des pratiques funéraires. Les professionnels sont nombreux à regretter le manque de concertation avec les pouvoirs publics. Du simple constat, à la sentence acrimonieuse, en passant par la dérision, les jugements sont sans appel. Il existerait un décalage entre les prescriptions, pour ne pas dire les préceptes, du législateur et le vécu des opérateurs. Pourtant, aucun consensus clair n'émerge suite à la lecture de ces différents avis. La profession est unie lorsqu'il s'agit de dire le manque d'attention qui lui est réservé, elle ne l'est pas à propos des contenus à défendre: «Ce que nous regrettons vivement et, ce décret en est l'illustration, c'est que les professionnels n'aient pas été entendus ${ }^{33}$; ou encore : "Troisième conclusion [et dernière, c'est nous qui insistons]: s'il y avait enfin une concertation réelle et approfondie (travail en commun) du ministère avec les professionnels, cela permettrait sans doute d'éviter ce genre de texte imprécis et mal adapté ${ }^{34}$. Nous laisserons sur ce thème le mot de la fin au directeur d'un

30. La phrase «ce décretne protégera quel'urne, etnon les cendres» étant reprise une seconde fois, plus avant dans le texte. T. Beaucourt, Président de l'Association pour les Droit et Obligations des Crématoriums.

31. P. Vidallet, Président de l'Union des Gestionnaires de Crématoriums Français.

32. Cela signifie que cette remarque est vrai: pour ceux qui voient dans ce texte un progrès, ceux qui sont pour le maintient d'un statu quo et ceux qui souhaitent que la proposition de loi votée au sénat soit de nouveau examinée.

33. P. Vidallet, Président de l'Union des Gestionnaires de Crématoriums Français.

34. F. Michaud-Nérard, Directeur Général des Services Funéraires Ville de Paris. 
crématorium, qui assume pleinement une vision du monde où les professionnels savent, puisqu'ils œuvrent en direct, et où le législateur devrait prendre acte de cette activité au quotidien avant de prendre toute décision. Incontestablement, cet avis est partagé unanimement par la profession, même s'il est habituellement présenté de façon plus lisse, comme une défense du pragmatisme. La distinction opérée entre ceux qui écrivent ${ }^{35}$ [les textes de lois, décrets...] et ceux qui vivent les situations et règlent les authentiques problèmes est nette: "Pourquoi les gens qui écrivent des décrets ne nous ont pas consultés? Nous aurions pu leur montrer la réalité du quotidien et leur faire comprendre tous les problèmes que soulève ce nouveau décret qui manque de sens pratique et qui, par son manque de rigueur, va créer plus de problèmes que de solutions, laissant une fois de plus aux professionnels le soin de régler les vrais questions... $\gg^{36}$. Raisonner de la sorte suppose d'établir une ligne de partage entre les professionnels et les autres, manifestant qu'uniquement les premiers sont en mesure de percevoir les problèmes et de définir les axes d'amélioration. L'univers du funéraire se referme du même coup dans cet entre-soi de connaisseurs ou dit autrement d'experts.

La critique peut devenir raillerie est remettre en cause le bien fondé de la loi : "Et pour finir sur un aphorisme, la dérision prêtant toujours à sourire, et en souhaitant que nul ne s'en offusque: "dans un système où plus personne n'est foutu de la connaître ou de la comprendre, il devient particulièrement injuste que force reste à la Loi” " ${ }^{37}$; ou du pouvoir régalien : "Pour finir, méditons cette phrase: "Si grands que soient nos rois, ils ne sont que ce que nous sommes" ${ }^{38}$.

Il ne faut pas voir dans ces remarques la seule motivation organisationnelle, à savoir les conséquences du décret sur le travail au quotidien. Si en effet, la crainte d'un alourdissement des tâches, notamment administratives, est réelle, elle ne résume pas l'ensemble des réactions. L'existence de ce décret en ce qu'il est officiel et effectif permet à chacun de se positionner quant aux évolutions pressenties du secteur funéraire, tant qu'un point de vue éthique, que professionnel au sens large. Sans entrer dans le détail de chaque avis, puisque la présente analyse en compte treize, on peut noter quelques partitions assez nettes:

35. L'extrait suivant insiste sur la question de l'interprétation des textes: «Comme toujours dans le funéraire nous allons assister à des exégèses à perte de vue sur les phrases, les mots, les intentions connues ou dissimulées du texte. [...] Il serait temps que nous ayons, nous les professionnels, enfin le courage et la volonté d'agir pour que la France du Funéraire ne soit plus le royaume des baronnies judiciaires ». J.-P. Sirugue, Président de VF Assistance.

36. P. Cave, Directeur du crématorium et du parc mémorial de la Vienne.

37. J.-P. Sirugue, Président de VF Assistance.

38. G. Véclin, France Obsèques Liberté. 
- certains, notamment les associations crématistes, sont favorables à un statu quo, à savoir que chacun soit libre de choisir ses funérailles et d'opter ensuite pour un lieu de sépulture ou non. La Fédération Française de Crémation par l'intermédiaire de son Président, fait savoir que ce décret est un moindre mal car, de ce point de vue, la proposition de loi du 22 juin 2006 remettait en question les principes même de la crémation à savoir «la reconnaissance expresse de la volonté du défunt» et «la libre disposition des cendres » (notamment à titre privé). Ce décret met à jour la prégnance du testament crématiste, qui selon la fédération devrait être renforcé ${ }^{39}$. Pour autant, J. Cousin rappelle que les crématistes qu'il représente n'ont « rien sollicité en la matière et le pseudo vide juridique ne nous gênait en rien; le respect dû au défunt ne se décrétant pas ${ }^{40}$. L'idée est que chacun doit œuvrer pour le respect des défunts et que celui-ci ne se décrète pas ${ }^{41}$. Cette conviction est partagée par des professionnels, même si elle est davantage manifeste chez les crématistes.

- certains, se félicitent de cette avancée marquant un juste équilibre. La métaphore de la balance est maintes-fois évoquée: «ce décret est donc, ànotre avis, un juste équilibre entre la liberté totale et le respect des cendres $\gg^{42}$, ou encore «Le ministre a tranché pour ne retenir que la question du devenir des cendres. Décision qui nous paraît équilibrée: la liberté des familles subsiste quant à la destination qu'elles souhaitent leur donner mais une responsabilisation individuelle est néanmoins instaurée par la déclaration préalable ou au dépôt de l'urne dans une propriété privée » ${ }^{\mathbf{4 3}}$. De ce point de vue, il fallait prendre acte qu'un problème de société se faisait jour et trouver des solutions afin que le respect dû aux cendres soit un droit.

39. Sur ce point, d'autres y voient l'occasion de développer un produit commercial, le contrat d'obsèques, qui a valeur testamentaire. La nécessité de mentionner de son vivant ses volontés, exprimée de la sorte dans le décret n²007-328 du 12 mars 2007: «Toutefois, si telle est la volonté exprimée par le défunt, soit l'urne est déposée ou inhumée dans une propriété privée, soit les cendres sont dispersées en pleine nature», devient une occasion de militer pour le développement des contrats d'obsèques. Cet aspect est le plus souvent suggéré, ou explicite dans ce cas: "Enfin, il constitue une incitation formidable pour toute la profession, à développer la commercialisation de contrats pré obsèques proposés en magasin. Les contrats vendus en magasins permettent en effet aisément de prévoir les volontés exprimées par les clients dans le détail, et de connaître ainsi leur souhait en matière de disposition de leurs cendres après leur mort », P. Gentil, Président du groupe ROC-ECLERC. On ne peut être plus clair.

40. J. Cousin, Président de la Fédération Française de Crémation.

41. D'où cette inquiétude: "L'avenir nous dira ce que réserve, en pratique, l'application de ce décret, mais il nous semble que ce dernier n'est que le reflet (éphémère?) d'un instant et que, dans quelques mois, une nouvelle session parlementaire risque de faire ressurgir d'autres velléités de réglementation et de législation bien dans l'air du temps. A suivre...», J. Cousin, Président de la Fédération Française de Crémation.

42. M. Marchetti et M. Minard, Co-Directeur de la Confédération des Professionnels du Funéraire et de la Marbrerie.

43. G. Véclin, de France Obsèques Liberté. 
- d'autres, souhaiteraient que les réformes soient plus massives. Il serait alors possible de penser que celles-ci pourraient s'inscrire dans le cadre d'une Loi cadre, comme la proposition de loi du 22 juin 2006 l'avait impulsé. Or, cela n'est pas si simple. Car, c'est dans un tout autre environnement que la demande de changement se tisse. Il s'agit avant tout de modifier l'organisation de la profession et plus largement du secteur funéraire dans son ensemble, étape incontournable avant même des changements plus massifs: «Va-t-on continuer à laisser représenter cette profession par d'autres qu'ellemême (...) Ou bien la profession va-t-elle prendre conscience, que parmoment il faut parler d'une seule voix, réunis et regroupés autour du même thème fédérateur $»^{\mathbf{4}}$. Bien évidemment, cela ne constitue pas une surprise dans la mesure où les avis ici examinés sont ceux de «professionnels » au sens large, non de juristes ou d'administratifs. Cependant, outre les divergences internes, c'est toujours la même ligne de fracture qui est mobilisée de façon archétypale: "A vous les organisations professionnelles une fois pour toutes, réunissez-vous sur l'essentiel (...) et proposez, proposez avec réalisme, mais tous réunis et non pas en ordre dispersé au gré d'intérêts particuliers. (...) A vous Messieurs les législateurs, trop de lois, trop de règlements sont finalement préjudiciables à tous car celui qui n'a pas un "navigateur" finit par se perdre $\gg^{45}$.

Comme nous l'avons indiqué en préalable, ces avis ne sont pas représentatifs de l'ensemble des points de vue, en outre, il va de soi que les intérêts défendus ne sont pas analogues. Néanmoins, leur analyse permet de mieux cerner le décalage qui peut exister entre un texte légal et l'appréciation qu'en ont les différents acteurs. D'autant que sur le terrain, là où les funérailles se font, c'est-à-dire dans les officines ${ }^{46}$, les ajustements sont encore légions. L'intérêt que nous portons à ces différentes mises à l'épreuve n'est pas, nous le rappelons, d'expérimenter la légitimité des textes et actions conduites, mais bien de voir en quoi ils participent de l'élaboration d'un système normatif qui vient dire, plus précisément chaque jour, ce qu'est la crémation et ce que doivent être les pratiques crématistes. Le discours maintes fois entendu que ce sont «les familles qui décident» doit être amendé, puisque les acteurs institutionnels et professionnels ne sont pas simplement des réceptacles qui viendraient faire la synthèse des initiatives individuelles, ou les accompagner.

44. G. Véclin, de France Obsèques Liberté.

45. J.-P. Sirugue, Président de VF Assistance.

46. Le secteur funéraire en France est composite. La part de l'activité artisanale est non négligeable, puisque $80 \%$ des entreprises compteraient moins de 10 salariés, et $58 \%$ moins de 5 salariés. Face, à cette activité, l'émergence de grands groupes est notable. Voir P. Trompette et S. Caroly, «En aparté avec les morts... Peur, larmes et rire au travail: les métiers du funéraire», Terrain, $\mathrm{n}^{\circ} 43$, septembre 2004, p. 68. 


\section{SUR LE TERRAIN...}

Face à l'évolution des textes légaux et aux débats qui animent la profession, les opérateurs funéraires sur le terrain, eux, n'ont guère changé leurs habitudes. Ayant interrogé une dizaine d'entre-eux ${ }^{47}$, nous avons abordé le thème de la crémation. Partant des expériences quotidiennes des assistants funéraires, le thème de la destination des cendres, de leur statut est évoqué, sans jamais faire directement appel au décret qui vient d'être pris. C'est à l'enquêteur de faire la démarche d'évoquer ce nouveau texte réglementaire. En effet, sa mention ne vient pas spontanément et nombre d'entreprises sont encore dans le flou à son sujet. Avant de rentrer dans le détail des pratiques professionnelles, il est significatif de noter que dans les faits trois attitudes sont à noter actuellement :

- soit aucune mention des transformations actuelles (dispositions prises par le décret) n'est faite à la famille. Les formalités administratives ne changent pas et l'opérateur, s'il s'enquiert de la destination des cendres en cas de crémation, c'est davantage pour savoir quelles prestations il va devoir réaliser en tant qu'opérateur (demande d'inhumation en columbarium, dispersion dans le jardin du souvenir, remise des cendres à la famille) que pour connaître le sort réel des cendres;

- soit il est fait mention aux proches qu'ils doivent faire une déclaration en mairie, l'assistant funéraire donnant un document à cet effet afin de faciliter la démarche auprès de l'administration ; une variante est davantage réglementaire car le document précise les conditions du décret dans le but que l'entreprise soit couverte en cas de problème, sans pour autant aiguiller la famille (il s'agit alors d'une précaution, non d'un conseil);

- soit encore, l'entreprise de pompes funèbres fait elle-même la démarche auprès de la mairie. Visiblement, il s'agit encore d'une position de principe, car il semblerait que les municipalités n'aient pas encore instauré de registre à cet effet. En cela, les professionnels anticipent les dispositions à venir.

En tout état de cause, on sent actuellement un flottement qui pousse les entreprises franchisées à attendre les directives de leur maison mère, et le plus grand nombre à prendre acte qu'il faut garder une trace en archives de la destination probable des cendres. Car, il nous a été dit à de nombreuses reprises qu'après tout, il est impossible de vérifier ce qu'il advient des urnes lorsque celles-ci sont remises à la famille: «Et oui! [embarras]

47. Sous la forme d'entretiens semi-directifs menés auprès d'assistants funéraires et de thanatopracteurs en juin 2007. L'enquête étant en cours nous nous baserons ici sur une dizaine d'entretiens ayant duré de $1 \mathrm{~h} 30$ à 3 h00. Lesquels ont été réalisés à Saint-Etienne et dans sa région. Par la suite, nous comptons étendre ce terrain à la région lyonnaise. 
Seulement ce qui se passe, à partir du moment où l'on remet l'urne à l'enfant, euh..., euh... Ben déjà après on peut pas faire la police derrière... et puis euh... l'urne elle n'a pas de valeur légale...» (Antoine).

Outre, cet état d'esprit qui pousse à assurer ses arrières, sachant que les dispositions légales sont nombreuses en matière funéraire, les professionnels sur le terrain ont tous un avis sur la question, au-delà du cadre administratif qui leur paraît toujours plus contraignant. C'est au détour d'expériences, de cas atypiques, de réflexions générales que la question des cendres et de leur destination est évoquée.

Lors d'un décès ou de la signature d'un contrat d'obsèques la destination des cendres est soulevée de manière tangible, si bien sur le choix a été fait de recourir à une crémation. Comme le dit cette assistante funéraire, les proches n'ont pas nécessairement fait la démarche de se questionner sur ce qu'il va advenir des cendres: "Je pose la question car il faut que je sache où vont les cendres et la généralement personne n'y a vraiment réfléchi, et bon ben..., ils me disent: "Qu'est ce que vous nous proposez"?» (Natacha). Opter pour la crémation ne suppose donc pas que des dispositions aient été prises en ce qui concerne le devenir des cendres. Certes, celles-ci n'ont pas de support légal mais elles ont une matérialité indéniable, bien différente du corps, mais tout de même.

Les réponses qui sont données aiguillent d'une certaine manière les vivants amenés à prendre en charge les restes de leur défunt. Elles nous ont surprises à double titre :

- d'une part, les possibilités sont présentées du point de vue du professionnel, à savoir du côté de son activité et non du côté du rapport entre les proches et le défunt (le souvenir de celui-ci);

- d'autre part, le terme d'inhumation est utilisé massivement sans rendre compte de l'opération qu'il implique étymologiquement. La symbolique déborde l'acte en lui-même, signifiant de la sorte que le pragmatisme sans cesse évoqué n'est pas exclusif.

Pour être clair, reprenons les extraits d'entretiens sur ces deux aspects. Plusieurs professionnels ont défini leur mission en matière d'information sur la destination des cendres de la manière suivante: "Et bien il y a trois possibilités: inhumation dans une tombe ou un caveau de famille; dispersion au jardin du souvenir; ou dispersion ailleurs » (Natacha). Ou encore: «Ben les possibilités sur la crémation, déjà leur dire, bon ben... Les lieux de destination des cendres : l'inhumation en concessions traditionnelles, les cases de columbarium, euh... La dispersion dans les jardins du souvenir ou dans des lieux privés. En général, c'est ces quatre grandes lignes... Éventuellement scellement sur les concessions aussi, puisque cela arrive à se faire» (André). Quand je demande à mon tour s'il n'y a pas d'autres possibilités 
c'est une réponse sous forme de silence, ou des cas inhabituels, forts rares qui sont mentionnés: "On a le cas d'une urne qui peut contenir les cendres de deux défunts» (Antoine); "J'ai assisté à des mélanges de cendres, le premier était décédé peut-être deux trois ans avant et ils ont mélangé... Enfin c'est la famille qui s'en est occupée »(Gilbert). Le dépôt de l'urne «à domicile» n'est jamais mentionné.

L'échange que j'ai eu avec ce jeune employé d'une entreprise familiale est tout à fait caractéristique. Il montre bien que le dépôt à domicile n'est pas l'affaire des professionnels, ils n'ont aucun droit de regard dessus et n'implique aucune action de leur part. Ce mode de conservation n'impute pas leur activité : il n'y a pas de demande de concession à faire, il n'y a de monument à réaliser, il n'y a qu'une remise de l'urne à effectuer au sortir du crématorium. Mais pourquoi alors traiter de la dispersion dans un cadre «privatif» (le plus souvent c'est la dispersion «en pleine nature» qui est évoquée, non la dispersion dans une propriété privée), sans évoquer la conservation «privative». Sans doute, parce qu'en matière de conservation, c'est le modèle de l'inhumation qui prédomine comme nous allons le voir dans quelques instants. Dès lors, l'oubli est total - simple omission, posture idéologique, désintérêt, ou attitude professionnelle - dans le sens où cette disposition discutée par le Droit (mais encore effective et légale) n'est pas même mentionnée par la profession. Voici, la tonalité de cet échange : "Vous n'êtes pas la seule personne à m'avoir dit qu'il existait ces différentes possibilités. Et celle qui consiste à garder les cendres chez soi? Oui, c'est une autre possibilité! (sourire avec une once de gêne). Mais, euh... (silence). Pour l'instant c'est possible. Oui, pour l'instant c'est possible, mais euh, pour l'instant je l'inclus entre guillemet dans la possibilité de la dispersion. C'est-à-dire, ou c'est inhumé donc dans ce cas là, la famille a pas... Donc si on inhume la famille n'a pas les cendres entre la crémation et l'inhumation, si on disperse non plus, la famille n'a pas les cendres entre le moment de la crémation et de la dispersion au jardin du souvenir. Par contre, quand on note "cendres remises à la famille", soit ensuite la famille les disperse, soit ils en font ce qu'ils en veulent. Donc dans ce cas là je leur dis: "vous les dispersez à tel endroit...", voilà et quelquefois "non, non on va pas les disperser on va les garder". Bon! Et vous ne dites rien? Non. Ça dépend... Non. Non parce que là ce serait vraiment mon avis, ce que je pense... Et je dois pas! J'ai pas à leur dire que je trouve que c'est pas du tout bien ou sain de garder "papy sur la cheminée”. Même si les cendres cela m'évoque rien, puisqu'il y en a derrière [à l'arrière du magasin]. C'est pas... Bon après...» (Matthieu). Ce discours n'est pas incohérent comme il pourrait le laisser paraître dans un premier temps, il marque simplement l'embarras de cet assistant funéraire à se positionner sur une activité qui pour lui ne relève pas de son cadre professionnel. 
L'autre surprise, comme nous l'avons mentionné plus avant, fut cet amalgame entre scellement sur un monument, dépôt dans une case de columbarium, et le terme d'inhumation. Sans entrer dans le détail de l'étymologie de ce terme qui nécessiterait une étude à lui seul, il est attesté que la référence à la terre, l'humus est constitutif de l'action d'inhumer qui suppose de «mettre en terre » et plus précisément «d'enfouir, de recouvrir de terre ». L'enterrement, la sépulture ont des sens approchant, rien de tout cela dans la crémation. Pourtant ! Indubitablement, une urne peut être inhumée, mais pour cela il faudrait qu'elle soit placée sous une couche de terre, le plus souvent dans un cimetière ou un site cinéraire. Pourquoi parler alors dans le cas de la conservation des cendres, du dépôt de celles-ci, d'inhumation sans restriction. Les motifs de cet effet de présentation sont langagiers mais pas seulement. Ils indiquent que le modèle de l'inhumation est débordant (puisqu'il quitte les sépultures classiques), mais qu'en parallèle il reste le modèle fondateur d'une culture en mouvement mais attachée à la culture de la trace et de la localisation des morts. "On parle d'inhumation dans un columbarium?... Oui... Oui, "inhumation d'une urne”... Oui. Mise en place d'une urne... Mais on parle plus d'inhumation. Oui...C'est vrai que c'est notre... (sourire). Disons que l'on doit à la fois garder..., On parlait du langage, on doit à la fois garder des termes assez simples parce qu'il faut qu'on soit compris par les familles. Il faut que les familles comprennent, il faut qu'on soit totalement transparent. Il faut qu'elles comprennent le pourquoi du comment on fait les choses. Le pourquoi du comment la loi nous oblige à faire ça. Et à la fois on doit aussi utiliser ce langage qui est particulier à notre profession où on doit à la fois appeler un chat un chat, mais où on doit avoir énormément de tact. Donc c'est... Une inhumation d'urne. C'est-à-dire que l'on gardera quand même le mot inhumation qui est quand même assez doux au langage, qui passera bien. Mise en place, euh..., Ça veut dire on met une caisse. C'est voilà! (Antoine). De la sorte, l'acte reste symbolique, alors que la portée d'un dépôt, d'une mise en place apparaissent quant à eux bien fonctionnels. Cependant, l'effet direct est de renoncer à une part de la spécificité de la crémation qui, de la sorte, devient d'une certaine manière une variante de la pratique inhumatoire.

Au final, la destination des cendres fait-elle réellement débat chez les assistants funéraires, au même titre qu'elle est objet de controverse pour le législateur, les pouvoirs publics et les organisations professionnelles? Le terme de débat est finalement inadapté ici, puisque dans un cas le débat est stoppé net car l'alternative d'une conservation "privée » est d'emblée désavouée et réduite au silence; dans l'autre, celui du «jardin du souvenir », les propos sont assez rudes chez certains professionnels, sans que cela donne lieu à discussion. C'est un avis, un avis définitif qui oscille entre sen- 
timent personnel (opinion) et posture professionnelle (avis étayé) qui anime certains professionnels ${ }^{48}$.

A ce titre, quelques assistants funéraires n'hésitent pas à interpeller toute personne, amis ou clients, qui souhaiterait voir ses cendres dispersées dans le «jardin du souvenir ». Cela peut-être dit lors de discussions informelles, mais également lors de la signature de contrats d'obsèques. La question peut-être également évoquée lors d'un décès, même si la façon d'énoncer son point de vue se fera plus feutrée, en raison de la situation qui ne suppose aucun retour en arrière. Point de mots assez durs pour qualifier le lieu: "Qu'est-ce que c'est un jardin du souvenir?”. Donc je leur explique. Alors là je donne un peu mon avis. "C'est très impersonnel". C'est sûr qu'il y a besoin d'un lieu, un lieu de mémoire, tout ce que l'on veut mais euh, pff, moi le jardin du souvenir, je suis pas... Je trouve ça triste. Je trouve çà très triste d'être avec tout un tas de personne. Oui... Avec en règle générale c'est les personnes âgées qui ont plus trop de famille. On décide alors qu'elles vont au jardin du souvenir» (Natacha). Le point de vue de Robert est tout aussi marqué. S'il repose sur une posture relativement similaire sur la place de l'individu, sur l'environnement et le testament crématiste, il apporte en plus une réflexion sur la dimension mémorielle de la sépulture. Proche de la retraite, ses propos sont emprunts du temps qui passe...: «La crémation c'est plus simple, c'est très fort. Moi qui suis un crématiste convaincu, je SUis CREMATISTE, membre d'une association et bien je considère qu'il faut penser à la destination des cendres. Les gens qui me disent: "ils ne viendront pas sur ma tombe”, pour pas déranger, en raison de l'évolution de la famille, et bien ils oublient que parfois, même une fois dans sa vie, quand on vieillit et bien on a besoin de se souvenir! Quand on repense à ses sources, à son enfance et bien on a plus de lieu pour se recueillir, c'est un problème. On rend pas service. Même pour une fois. Il y a un devoir de mémoire. Il vautmieux procéder à l'inhumation des urnes. Je dis souvent, ceux qui veulent se faire incinérer, ils veulent un peu se démarquer, alors le jardin du souvenir, c'est disperser ses cendres avec les autres. Alors qu'on aime pas trop se mélanger aux autres, ... On veut être différent » (Robert).

Le leitmotiv de la discrétion, du respect des volontés du défunt comme de ses proches, est une donnée que l'on ne peut évacuer. Cependant, les professionnels s'ils ne se positionnent pas directement sur les termes du débat tels qu'ils ont pu être formulés au Sénat lors du vote de la proposition de loi du 22 juin 2006, ou même en réaction au décret du 12 mars 2007, ont des attitudes aussi bien formelles que langagières, quasi-journalières, qui

48. Cet aspect n'étant pas traité par le législateur, pas plus que les organisations professionnelles. 
impactent directement les pratiques et représentations de l'acte crématiste.

A leur manière, ils posent un cadre tout aussi effectif que ne le font la loi et les textes légaux. Moins visible, puisque travaillé touche par touche et non l'objet de revendication, ce travail au quotidien participe de l'élaboration d'une norme au sujet de la crémation. Norme qui donne des « directions » quant à la manière d'appréhender les cendres, de les localiser, de les conserver ou non, mais bien plus que cela qui redéfinit - comme l'appelait de ces vœux la proposition de loi du 22 juin 2006 - l'univers même du funéraire. Penser la crémation, la doter de règles, de valeurs, de rituels... Suppose de repenser de concert l'inhumation.

Malgré toutes les dissensions recensées dans cet article, reste un point d'ancrage, commun au législateur, aux organisations professionnelles, aux opérateurs funéraires. Personne ne discute le bien fondé, à l'exception peutêtre des crématistes de la première heure, que le cimetière devienne le référent quasi-unique en matière funéraire et plus précisément de sépultures. Une sorte de naturalisation de la règle émane des discours, attestant qu'un processus de normalisation est à l'œuvre. Il va de soi..., Il est naturel... Que le cimetière devienne le cadre idéal pour accueillir tous les morts et permettre aux vivants de se rassembler dans un lieu, public, ouvert à tous. Comme s'il en avait toujours été ainsi ! Comme s'il n'y avait pas d'enjeux économiques, idéologiques?

Parler en termes juridiques comme le fait G. D'Abbadie, "la volonté de mieux encadrer la destination des cendres par le pouvoir réglementaire, en suggérant que le cimetière public est bien le lieu de droit commun de la destination des cendres (...) ${ }^{49}$, avec toute la nuance que cela suppose, est le premier niveau d'interprétation du texte. Un deuxième niveau consiste à inverser les termes de la proposition: "l'inhumation ou le dépôt ou la dispersion des cendres dans un cimetière ou dans un site cinéraire, c'est-à-dire dans un lieu ouvert au public, devient la règle $»^{50}$. Enfin, une troisième et dernière étape n'énonce plus les conditions de l'élaboration de la règle pour la faire advenir comme normale, évidente, inaliénable. Le dessein est tout à fait différent et le contexte (celui du décret) peut ensuite être rayé sans dommage: "Le cimetière devient le lieu naturel de destination des cendres... ${ }^{51}$, "Heureusement, cette nouvelle version présente bien le cimetière ou le site cinéraire comme la destination naturelle des cendres $»^{\mathbf{5 2}}$.

49. G. d'Abbadie, Co-auteur du Code pratique des opérations funéraires. Lequel réagissait ici, au même titre que les autres interlocuteurs au décret du 12 mars 2007.

50. C. Loiodice, Présidente de l'Union des Professionnels du Pôle Funéraire Public.

51. «... Ce qui ne peut qu'être favorable aux entreprises ». Naturel ou enjeu économique? M. Marchetti et M. Minard, Co-Directeur de la Confédération des Professionnels du Funéraire et de la Marbrerie.

52. F. Michaud-Nérard, Directeur Général des Services Funéraires Ville de Paris. 
Le passage de la règle à une norme intégrée et non discutée est central pour comprendre les déplacements qui s'opèrent aujourd'hui. Dans le cas de l'inhumation le cimetière est culturellement et réglementairement le seul lieu de référence en matière de sépultures. Dans le cas de la crémation rien ne stipulait jusqu'à l'heure qu'il en était de même. La proposition de loi du 22 juin 2006 visait en partie à entériner le fait que dans un «esprit républicain, laïc et public », il fallait redonner sa place au cimetière afin que chacun puisse se recueillir sur la sépulture d'un défunt et que le culte du souvenir demeure. Le décret du 12 mars 2007, s'est positionné quant à lui de manière administrative, tout en conservant l'idée sans vraiment la mentionner qu'il y aurait une façon de procéder, usuelle, banale, ordinaire, en un sens «normale » et une exception à la règle mentionnée par cette incise: «Toutefois $»^{53}$. Ainsi, sans volonté de se démarquer de la part «du défunt», sans l'effort de la mention de cette volonté, les cendres seront conservées ou dispersées dans un cimetière ou site cinéraire.

Une page est ainsi en train de s'écrire, une page capitale de la vie en société, puisqu'elle implique davantage que les morts.

\author{
Gaëlle CLAVANDIER \\ Maître de conférences \\ Université de Lyon \\ Université Jean Monnet Saint-Etienne \\ MODYS, UMR CNRS 5264 \\ Gaelle.Clavandier@univ-st-etienne.fr
}

\begin{abstract}
RÉSUMÉ
La place de la crémation, aujourd'hui en France, est pérenne. Cette assise est perceptible à partir du moment où tout un chacun réfléchi quant au mode de sépulture qui sera le sien. Sans remettre en cause l'inhumation, l'évolution des pratiques et des mentalités à l'égard de la crémation, implique de redéfinir l'univers du funéraire. Expérience originale voire marginale, les pratiques crématistes sont assimilées à des comportements ordinaires. Malgré, tout, émerge çà et là l'idée d'un nécessaire débat sur les conditions même de l'acte crématiste mais plus généralement sur son environnement: quel statut conférer aux cendres? Comment peut s'établir un souvenir «des morts» dans ce contexte ? Le principe d'un lieu de recueillement public est-il remis en cause? Faut-il que la personne qui a fait le choix d'être crématisée le déclare, attestant de la sorte que le caractère usuel de la pratique n'est pas aussi évident
\end{abstract}

53. «Toutefois, si telle est la volonté exprimée par le défunt, soit l'urne est déposée ou inhumée...», additif à l'article R. 2213-39 du Code général des collectivités territoriales. 
qu'il n'y paraît? L'hypothèse que nous faisons, est que depuis les années 2000 se met en place une réflexion qui définit «ce que doit être la crémation »; laquelle se travaille d'un point de vue institutionnel, mais aussi sur le terrain, là où se fait la prise en charge des funérailles. Une nouvelle norme est en émergence qui vise à donner un cadre réglementaire, à aiguiller les pratiques et à les asseoir sur un système de valeurs et de croyances, la norme établissant le normal. Cet article portera plus précisément sur les « réactions » des professionnels suite au décret du 12 mars 2007 concernant «la protection des cendres », avec deux terrains: les avis des représentants des organisations professionnelles, associations et syndicats ; les actions et conseils au quotidien des assistants funéraires. 